Viharos, Zs. J.; Chetverikov, D.; Háry, A.; Sághegyi, R.; Barta, A.; Zalányi, L. Pomozi, I.; Soós, Sz.; Kövér, Zs.; Varjú, B.: Vision

\title{
Vision based, statistical learning system for fault recognition in industrial assembly environment
}

\author{
First Zs. J. Viharos, Member, IEEE; Second D. Chetverikov; Third A. Háry; Fourth R. Sághegyi; Fifth
}

A. Barta; Sixth L. Zalányi; Seventh I. Pomozi; Eighth Sz. Soós; Ninth Zs. Kövér and Tenth B. Varjú,

\begin{abstract}
The paper presents a statistical learning system based visual solution developed and applied for fault detection in industrial environment. As a mobile vision system the area of use was the automatic detection of rare faults in complex assembled objects. The object detection, the fore- and background separation, and the multimodel database enables the system to manage irregular batches of the different objects. A multi-model database guarantees that the object is compared with the statistically most relevant model, therefore it reduces the number of false alarms. The developed system is able to detect faults with the size of $2 \%$ of the total picture based on previously learned models.
\end{abstract}

Index Terms - Computer vision, image analysis, learning system, model-based vision, pattern recognition, vision for industrial assemblies.

\section{INTRODUCTION}

Product quality is always a primary concern in industry. In mass production the manufacturers must protect the customers from receiving a defective product. Before starting a new technology or product a risk evaluation should highlight the possible defects.

On the assembly lines most of possible defects are visually noticeable: swapping parts, not properly positioned parts or possible damages. These failures indicate a high amount of visual system on production lines, each taught for dedicated "known" failure mode.

F. Zs. J. Viharos is with the Institute from Computer Science and Control, Hungarian Academy of Sciences, Budapest, Hungary (e-mail: viharos.zsolt@sztaki.mta.hu)

S. D. Chetrverikov is with the Department of Informatics of the Eotvos Lorand University, Budapest, Hungary (e-mail csetverikov.dmitrij@sztaki.mta.hu).

T. A. Háry is with APNB Research, Service and Development Ltd., Zalaegerszeg, Hungary (email: andras.hary@apnb.hu)

F. R. Sághegyi is with Pannon Development Foundation, Technology Center, Zalaegerszeg, Hungary (email: ramona.saghegyi@tc.org.hu)

F. A. Barta is with Drem Ltd., Budapest, Hungary (email: andras.barta@drem.hu)

S. L. Zalányi is with Drem Ltd., Budapest, Hungary (email: zalanyi@drem.hu) and Wigner Research Centre for Physics of the Hungarian Academy of Sciences

S. I. Pomozi is with Drem Ltd., Budapest, Hungary (email: istvan.pomozi@drem.hu)

E. Sz. Soós is with Opel Szentgotthárd Kft., Szentgotthárd, Hungary (email: szilveszter.soos@gm.com)

N. Zs. Kövér is with Opel Szentgotthárd Kft., Szentgotthárd, Hungary (email: zsolt.kover@gm.com)

T. B. Varjú is with Opel Szentgotthárd Kft, Szentgotthárd, Hungary (email: balazs.varju@gm.com)
The processing algorithm is a pixel to pixel comparison: reading numbers or codes, detect edges, etc. However, sometimes new failure modes came up, which were judged as impossible or simply nobody thought they could happen. These failures can be grouped into one category: the "unknown, rare problems". They happened only once, but caused customer complaints. At the moment, there is no error detection system which can find these unique failures. This was the background where the idea of a learning vision system came from. The imagined system is not created to find exact failures, it should only review the products and check if they are all the same. The system has no connection to the assembly process, it only carries an external visual observation and indicates if products are not the same. In order to prepare a general vision based production supervision solution, the system should be mobile for easy relocation, and must work without any additional light source, only by using the illuminance of the environment. There is only visual connection to the line via the camera, also supporting the easy relocation. It should automatically detect the presence of the product and should deal with bothering movements in the background, too. With these conditions, all negative limitations of the commonly used vision systems could be eliminated.

The system should work as if someone would be standing next to the assembly line and watching the products. This person doesn't have to know the details about the way how the product works, just check the visual appearance and know that all of them should look the same. After the system is placed next to the production line only the camera needs to be adjusted in some basic visual observation parameters and the learning phase can begin. During this phase the user has to ensure that the first five or ten parts are good. When it's done, the system will also know that all the following parts should look the same. If one looks different it just indicates it and someone who knows the product will check if it is an error or an allowable difference.

Due to many customers with different demands, high component level proliferation is given. One of the most complicated function will be the handling of the different product types. In case it's only a small difference between two parts, it can be a failure, if the deviation is bigger or it can be a new type, however, sometimes a small difference can also mean a new type.

\section{STATE-OF-ART: ALGORITHMS AND INDUSTRIAL VISION}

The given fault detection task has a number of specific features which are not typical for traditional industrial inspection. With a reasonably low false alarm rate, the system has to signal very rarely occurring, unspecified assembly 
defects in unknown positions. This lack of fault model means that learning the potential faults is quite difficult since they appear just as "significant deviations" from the "normal" fault-free state that can be called "anomalies". As discussed in [1], such situation arises in inspection of modern highintegrity systems where standard classification techniques are not applicable. In addition, the solution has to cope with frequent visible but allowable deviations, which necessitate careful checking all major deviations.

Similar phenomena are studied in various sciences as well as in telecommunication, banking, business and security tasks, under different names: defect, anomaly, motion, change, novelty, unusual behaviour, unexpected event, abnormality. Despite the obvious similarity, however, finding a common mathematical background is not easy because different areas use different mathematical tools and perceive the problem in different ways $[1,2,3,4]$. Fortunately, there is a general understanding that the root of the problem is in detecting the so-called outliers $[3,4]$ that differ from a regular behaviour represented by some statistical model.

The principles of novelty detection and processing are discussed in [1]. A novelty is a phenomenon, event or data that does not fit the current model. When a novelty is detected, the system has to decide whether to accept it and update the model, or to discard it and add it to the set of negative examples or patterns. The criteria of a good novelty detection system include maximising the detection rate while minimising the false alarm rate. The appropriate normalisation of data, the small number of parameters to tune, the capability of generalisation without increasing the false alarm rate, the scalability, and the capability to work with small and noisy learning sets is required, too. Most of these criteria aim at the robustness of the system since the improperly handled outliers can spoil the model.

A critical related issue is that of rejecting a sample without decision making in case of uncertainty. Most of the defect recognition systems have a threshold parameter specifying the sensitivity of the system. The given application has to address this problem since the lack of decision may result in undetected faults. Here, an option is to delegate the decision to a human operator which is possible if such occasions are rare.

The survey [1] discusses a number of mathematical tools that can be used for novelty detection, including the parametric ones such as Gaussian Mixture Modelling (GMM) [5], Principle Component Analysis, Singular Value Decomposition (SVD) [6] and clustering; and the nonparametric ones such as k-nearest neighbours and Parzen windows. The paper [1] mentions also several industrial applications, e.g., inspection of aircraft engines using signal processing. Another relevant survey of industrial computer vision is provided in [7].

Change detection in images and videos [2] has numerous applications in different areas including industrial inspection. Despite its apparent simplicity, this problem is not easy at all as changes may appear due to many significant and insignificant factors such as object motion, shape variation, occlusion and discussion, illumination variation, shadows, camera motion or changing background. GMM [5] and SVD [6] are popular tools for background modelling [8,9] and change detection in case of static camera. These tools can be useful in the given industrial challenge, as well. However, special attention should be paid to irrelevant appearance changes of the inspected object because of noise, illumination changes, mapping distortions due to object motion, background variation and other practical issues.

In conclusion, the given fault detection task is a complex, challenging problem that needs careful planning, combination and application of sophisticated mathematical, programming and engineering tools.

There are a lot of visual recognition systems in the automotive industry for measuring, and analysing product components, for investigating the accuracy of the manufacturing process, checking the known part presence or absence, sorting products, or reading and verifying data mark. Some of them work with pixel-pixel comparison, with linetrigger, or use pre-trained reference pictures, or pre-set limits, but they do not work with complex parts, with irregular line sequence, or with model variance without user intervention or training.

Some paper deal with visual applications in industrial field, in connection with leaning-based and knowledge-based algorithms, but most of them investigate only position detection [10], or variety of the parts [11], but not complex assembly.

Woods el al. worked out a frame-based system for modelling a visual task, where the user has to program the system, but no vision specialist is required. This system implemented for several practical applications e.g. to chromosome classification, and brake assembly investigation for crack detection $[13,14]$, but it needs a user for operation, and cannot learn, and use previously acquired data.

As conclusion, no ready-made solution is available, which is able handle this complex vision task, so a novel solution was required to handle this supervision problem.

\section{HARDWARE SYSTEM AND REQUIREMENTS}

Parts of the hardware unit had to be selected carefully, to achieve real-time processing with approximately 30 second cycle time, while the algorithm has to solve many tasks: it has to detect the object from motion information, collect frame pictures from it, separate the object from the background, find the statistically most corresponding model with comparing the object to all stored models, and analyse the variance, using statistical methods, and finally, making the decision that the object is imperfect or not.

The system has to be mobile for the easy relocation, which means that it has to be independent from the assembly line, and exclude the usage of external light source, e.g. because it can disturb the operators.

The only information coming from the object is the motion, but it is not moving with constant speed, and not moving always (it can stop whenever the line is overloaded), and the background can also move often, because behind the monitored line is another assembly line, with similar moving objects, in another assembly status.

For an adequate image quality a 5 MP industrial camera was chosen. Finding the ideal camera objective was essential, because of the limited space in the production line area (approx. 1-3 meters). The selected camera objective provides 
the optimal fit to size of the image as well as the proper sharpness and brightness.

Additionally, a tripod with hydraulic damping and a warning system with both light and sound signals were integrated. These suit the industrial environment and keep the mobility of the system at the same time.

\section{ANALYSIS OF THE IMAGE}

According to every data analysis, the process starts with gathering the most available and relevant data. Noise originating from the capturing device is always present, the lighting conditions, shadows or slight changes in positions of the object relative to each other also usually happen. It was a requirement not to train the system in advance, and to reduce operator interactions to the minimum, so the system has to start up and operate automatically. The system needs to model the whole world from scratch, but it may learn adaptively, utilizing as many procedures as necessary to reach the desired response quality.

The information that could be used were that the products were rigid bodies, they moved with more or less constant velocity (on a production line) and the operator had to set up two frames giving some hints about the expected product size and possible position passing by on the production line.

Another constraint was formed by the lighting conditions: the factory was equipped with fluorescent lighting with a $50 \mathrm{~Hz}$ flickering frequency, and it was not allowed to use additional illumination because of labour safety reasons. The given low intensity illumination limited the video frame rate to a maximum of $10 \mathrm{fps}$.

Given the above described conditions, it was decided using the robust optical flow algorithm to track specific feature points in the video sequence (Fig. 1.).

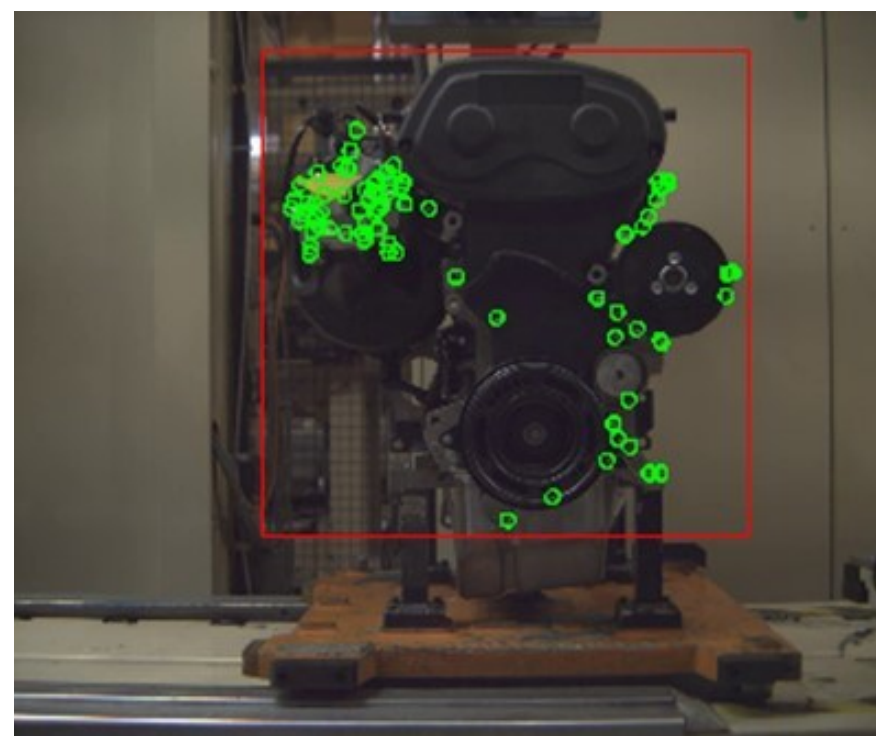

Fig. 1. Product (combustion engine) recognized on the production line by the optical flow of feature points (green circles) and the bounding box of the product (red rectangle).

As no predefined models were given, either from the background or form the product, a system was needed to collect a few data at the beginning, prior to the evaluation of possible defects. After a few products passed through a statistical image model can be built up for both of the background and the product. Lacking an apriori model of the product the same is needed for the products: separate them from the background, align the determined product image to the previously captured ones, and build a statistical model for it.

\section{FAILURE DETECTION ALGORITHM}

After the model for both the background and product has been established, a single detection procedure is working as follows: detecting characteristic feature points; filtering them based on the velocity constraints; if enough feature points found, draw a bounding box around them; pass these points to the optical flow algorithm to track them. To avoid mixing all these different product views into a single model, a few positions were fixed at which the captured images are analysed, and the models are built separately. The newly captured product image at a certain position is then aligned precisely to the product model at that position, and screened for differences.

In a general production environment, the background is not homogeneous, there might be other moving objects, workers, blinking/flickering lights behind the assembly line, and the color of parts of the background can be very similar to that of the product to be investigated. Obviously a black object cannot be detected in front of a similarly black background. Melting the masks from the different viewpoints yields the maximal consensus mask, which will cover the object at any position (Fig. 2.). The hypothesis was to neglect changing shadows, as they were rare, and in a specific position on the production line the object together with its shadows appeared typically in the same way.

The next step is the major goal of the solution: fault detection. Similar difficulties were faced to the object identification process: the lack of fault model or fault definition. In this case the situation was even worse it was required to detect faults that were rare and unusual in any sense, there was no typical size or position of the defects. In this case a statistical pixel based comparison could be performed as "novelty detection".

The defect recognition of very complex products, however, resulted in new difficulties. Parts of the product can be placed in different, equally acceptable positions: coloured wires in a cable harness or some moving or rotating parts of the product. After collecting enough data (evidence), the statistical model would accommodate to these circumstances locally. A single Gaussian model e.g. can handle such a situation for the price of loosing the sharp accuracy of the defect recognition locally. This approach opens the opportunity to apply unsupervised modelling techniques, like Principal Component Analysis (PCA). Unfortunately, PCA cannot be used in advance, as one might have catch such variability combinations in the first few products (and make a projection onto their hyper-plane).

Now the problem description is complete: without external intervention (fixing background or applying additional illumination) to the measured scene, lacking the model of background, and the model(s) of the products, furthermore lacking the model(s) of possible defects, the 
system should automatically build all these representations and utilize them to capture defects of the different products.

One question arises immediately: what distinguishes a defected item from a different type of product compared to the models of the previous products? The procedure is based on the heuristic assumption, that the errors are rare, and to get two or more basically identical but defective product images has a low probability. Still, it is unknown in advance, if a new type of product has arrived on the line or the regular one had a fault. In case of rigid bodies, the shape of the products can be compared supposing that exactly the same shape necessarily belongs to the same type of product. The effectivity of all these measures largely depends on the reliability of the underlying data, e.g. how precisely is possible to derive the same feature points in images of two identical items taking the changing environmental circumstances into account, too.

Once a procedure yielding acceptable product type separation is established, it allows to build individual statistical models for fault detection of each type, and also to further increase the quality of type separation.

\section{RESULTS}

In the tests of the system, carried out in the production line area, particular segments of the algorithm were being analysed during promoted special events.

Based on the test results, the system runs stable, the first phase thus accomplished, failures with the minimum size of $2 \%$ of the whole image were located. Other rarely occurring known failures were produced and detected which have no influence on the proper working, but have a risk later if repaired by the customer (Fig. 2.).

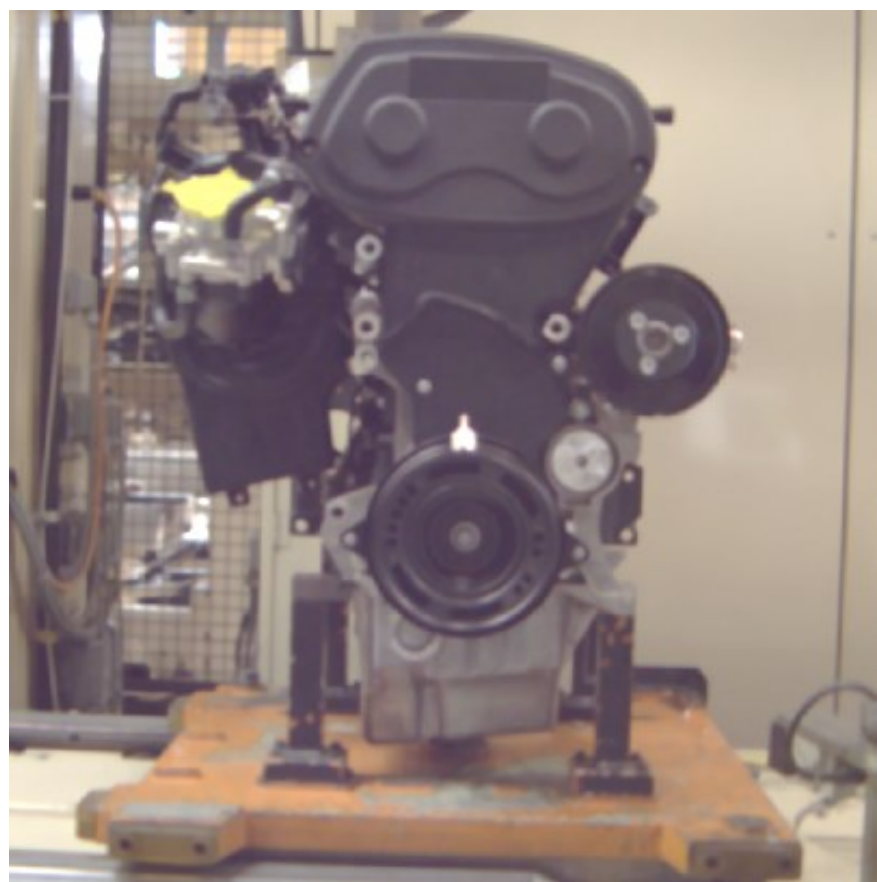

Fig. 2. Inducing a possible failure: a small key placed on a product.

The related software platform developed for the system is user friendly and easy to understand (Fig. 3.). Moreover, it makes possible to review a formerly detected fault, which may be just a change in the model, and if needed add them to the base model as an update, consequently, continuous knowledge extension is allowed.

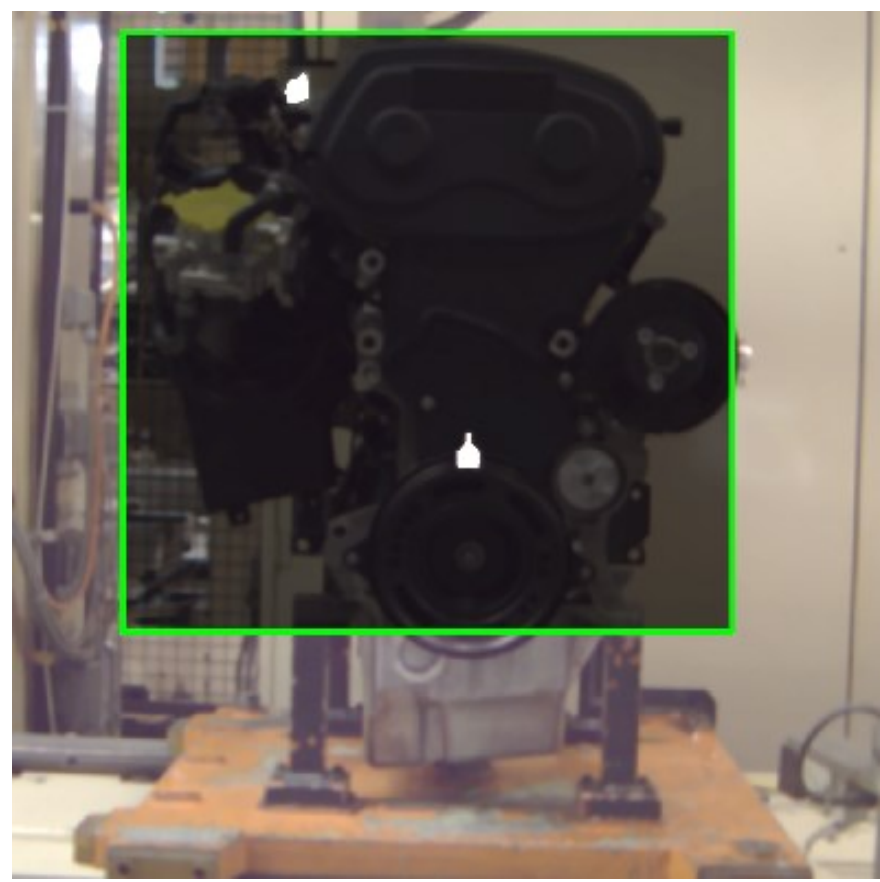

Fig. 3. Results of the fault detection, the key outlined in white.

\section{CONCLUSIONS}

The paper presented a vision based, statistical learning system for fault recognition in industrial assembly environment. The literature review of vision systems' applications in industrial quality control mirrored that many camera based solutions are applied in production plants but used typically for detect special, in advance prescribed failures that may occur frequently. The introduced novel solution is addressing the other segment of production failures that are not frequent and without pre-specification in advance. Another significant difference is in relation to the camera working conditions, the quality checking solution should work in changing lighting conditions, without any fixed position and without any field or product specific preliminary given knowledge, consequently learning capability was required. These expectations drove the research to discover and develop a generally novel solution for vision based industrial quality checking.

The system works as expected, and is reliable in fault detection, it requires minimal operator intervention, installation is quick and no expert skills are needed. Failures with the minimum size of $2 \%$ of the whole image were located and novel, promptly arising failure of such type is possible to detect with the proposed solution.

\section{ACKNOWLEDGEMENT}

Work presented here has been supported by the grants of the Highly Industrialised Region in Western Hungary with limited R\&D capacity: "Strengthening of the regional research competencies related to future-oriented 
manufacturing technologies and products of strategic industries by a research and development program carried out in comprehensive collaboration", under grant No. VKSZ_121-2013-0038.

\section{REFERENCES}

[1] M. Markou, S. Singh, "Novelty detection: a review-part 1: statistical approaches", Signal Processing, vol. 83, pp. 2C71-2497, 2003.

[2] R. Radke, S. Andra, O. Al-Kofahi, B. Roysam, "Image change detection algorithms: a systematic survey", IEEE Trans. Image Processing, vol. 3, pp. 294-307, 2005.

[C3] V. Chandola, A. Banerjee, V. Kumar, "Outlier detection: A survey", ACM Computing Surveys (CSUR), vol. C1, 2007.

[4] V.J. Hodge and J. Austin, "A survey of outlier detection methodologies", Artificial Intelligence Review, vol. 22, pp. 85-126, 2004.

[5] C. Stauffer, W. Grimson, "Learning patterns of activity using real-time tracking, IEEE Trans. Pattern Analysis and Machine Intelligence", vol. 22, pp. 747-757, 2000.

[6] F. Kahl, R. Hartley, V. Hilsenstein, "Novelty detection in image sequence with dynamic background", Springer LNCS, Germany, vol. C847, pp 117-128, 2004.

[7] E.N. Malamas et al., "A survey on industrial vision systems, applications and tools", Image and Vision Computing, vol. 21, pp.171-188, 2003.

[8] D. Hull, J. Nascimento, "Comparison of target detection algorithms using adaptive background models", Joint IEEE Workshop on Visual Surveillance and Performance Evaluation of Tracking and Surveillance, pp. 117-128, 2005.

[9] A. Sobral, A. Vacavant, "A comprehensive review of background subtraction algorithms evaluated with synthetic and real videos". Computer Vision and Image Understanding, vol. 122, pp. 4-21, 2014.

[10] H. Niemann, H. Brünig, R. Salzbrunn, and S. Schröder Knowledge-Based Vision System for Industrial Applications Lehrstuhl ffir Informatik 5 (Mustererkennung), Universitfit Erlangen-Nfirnberg, Martensstrage 3, 1990

[11] Masahiko Yachida And Saburo Tsuji, A Versatile Machine Vision System For Complex Industrial Parts, IEEE Transactions On Computers, VOL C-26, NO. 9, SEPTEMBER 1977

[13] Peter W Wodds, David Pycock, Cristopher J Taylor - A frame-based system for modelling and executing visual task, Images and Vision, 1989

[12] Woods, P W, Taylor, C J, Cooper, D H and Dixon, R N 'The use of geometric and grey-level models for industrial inspection' Patt. Rec. Letters Vol 5 (1987) p 11

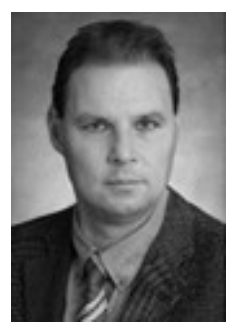

First Zs. J. Viharos (M' 2016) received his Ph.D in 2000. Currently He is working for the Institute from Computer Science and Control, Hungarian Academy of Sciences. His research interests cover the fields of sensor systems, signal processing, data mining, big data, learning machines, like artificial neural networks and neuro-fuzzy systems, numerical search and optimization algorithms, system decomposition, incomplete data handling, information theory, simulation, applied in various areas, like production systems, wind turbines and wind farms, traffic control and in many types of machining in order to realize solutions for technical monitoring and diagnostics, quality management, management reporting, numerical assignment solving and subsystem identification. Ha was publishing around 100 scientific papers resulting until now in around 300 references.

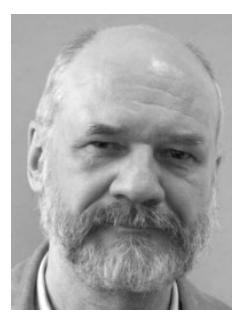

Second D. Chetverikov was born in 1952 . He studied theoretical physics at the Faculty of Physics of the Moscow State (Lomonosov) University, Russia. He received his $\mathrm{PhD}$ and $\mathrm{DSc}$ degrees from the Hungarian Academy of Sciences in 1988 and 2004, respectively.

Since 1978, he has been a researcher at the Institute of Computer Science and Control, Hungarian Academy of Sciences (MTA SZTAKI) Budapest, Hungary. Currently, he is a Professor at the Department of Informatics of the Eotvos Lorand University (ELTE) and a Scientific Adviser at SZTAKI. He is the author or co-authors of over 150 research papers. His research interests include computer vision, image and video processing and analysis, sensor fusion and scene reconstruction.

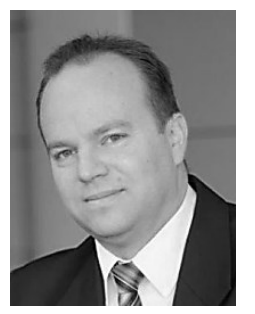

Third A. Hary MSc in Mechanical Engineering, MBA, PhD in Economics \& Management Sciences, all qualifications are from Budapest University of Technology and Economics. From 1998 to 2003 he worked for Philips in various management functions. From 2004 onwards, he is managing director of APNB Research, Service and Development Ltd. offering services in operational and business excellence, local economy development programs, R\&D programs and systems management. Acts as head of Zalaegerszeg Technology Center and co-ordinates city industrial \& economy development program as well as Pannon Mechatronics Cluster.

His research area is complex and partially structured systems with special attention to their cohesion behaviours as well as control features. 


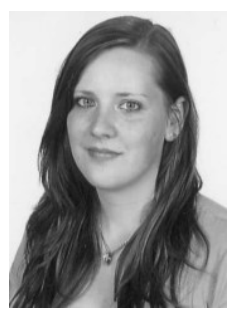

Fourth R. Sághegyi was born in Szombathely, Hungary in 1991. She received the BS degree in 2014 in mechatronical engineering at The University of West Hungary, Zalaegerszeg, Hungary.

Since 2011 she has been a development engineer, at Pannon Development Foundation, Technology Center.

From 2011 she participated in more then, 40 R\&D projects in main fields of design engineering, measuring and testing system development, material science and process development.

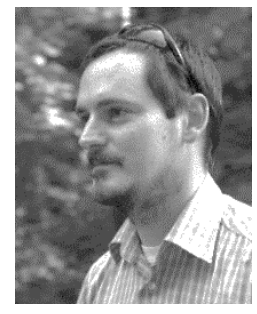

Fifth A. Barta was born in Budapest, Hungary in 1977. He received the MS degree in 2001 and the $\mathrm{PhD}$ degree in 2008, both in physics from the Eötvös University of Sciences, Budapest, Hungary. From 2000 he is the member of the Environmental Optics Laboratory of the Eötvös University. From 2004 he participated in several industrial development projects in design, control and image processing topics. He is the author of 43 peer reviewed scientific papers, 3 book chapters and 3 patents. His research interests include light polarization, environmental optics, biooptics, polarization vision.

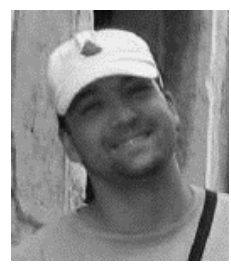

Sixth L. Zalányi was born in Budapest, Hungary in 1975. He received the MS degree in 1998 and the $\mathrm{PhD}$ degree in 2008, both in physics from the Eötvös University of Sciences, Budapest, Hungary

From 1998 he is first Research Assistant and now Research Fellow at the Wigner Reseach Centre for Physics of the Hungarian Academy of Sciences. From 2010 he participated in several industrial development projects in algorithm design for image processing topics. His research interests include machine learning, statistics, computational neuroscience and dynamical systems.

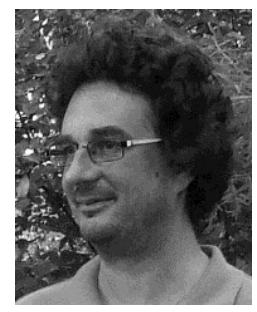

Seventh I. Pomozi was born in Budapest, Hungary in 1974. He received the MS degree in 1997 and the $\mathrm{PhD}$ degree in 2000, both in physics from the Eötvös University of Sciences, Budapest, Hungary. He is a former Senior Systems Designer at General Electric.

From 2000 he led several innovative industrial development projects in the topics of optical quality control in mass production, license plate recognition and automatic medical instruments. He is the author of 24 peer reviewed scientific papers and 3 patents. He has over 10 years experience in parking service business, computer vision, recognition research and optical applications in industrial environments.

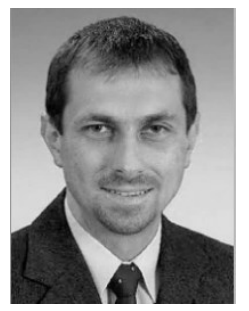

Eighth Sz. Soós received his BA degree in mechanical automation from Kecskemét College, Faculty of Mechanical Engineering and Automation in 1988.

In 1991, he started working at Opel Szentgotthard Ltd., where he performed in several functions during the years, including Maintenance, Manufacturing Engineering, Production and Quality. After a 2-year-long international assignment, he has been working as a Manufacturing Engineering Manager since 2013.

Mr. Soós actively participated in several R\&D projects from Opel side in collaboration with regional universities.

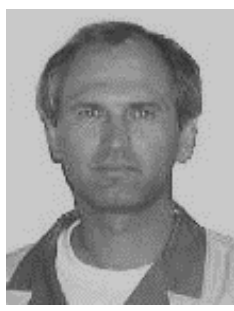

Ninth Zs. Kövér was born in Szombathely, Hungary, in 1964. He received the MSc degree in electrical engineering from the Budapest University of Technology. From 1989 to 1991 he worked in developments of digital circuits and controls in company Signelit. From 1991 he worked for General Motors in several areas: he was ME engineer in car assembly plant, test engineer in the Continuously Variable Transmission plant and worked as Shainin / Red X plant master in technical problem solvings at Quality department. His first local publication is a Lessons Learned Handbook of technical problem solving for powertrains. Beyond the job his research interest as a glider flight instructor is the human error reduction in recreational aviation. This same human error is responsible most for nonconform products in the assembly businesses. He initiated the first version of vision project in 2009 .

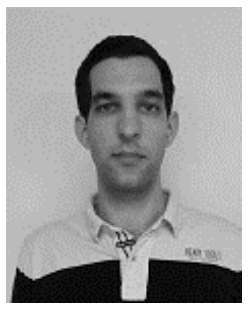

Tenth B. Varju received his BA degree in electronic engineering from Széchenyi István University, Faculty of Automation in 2009 and his MA degree in electronic engineering at the same university in 2011.

In 2011, he started working at Opel Szentgotthard Ltd. as a Manufacturing Engineer of an assembly line. In 2013, he became the test engineer of the same line. From 2016 he is responsible for new project integration on assembly lines.

Mr. Varju has been participating in a few R\&D projects from Opel side in collaboration with regional universities since 2012. 